\title{
Localized Lesion of Caudal Part of Lobus Parolfactorius Caused Impulsive Choice in the Domestic Chick: Evolutionarily Conserved Function of Ventral Striatum
}

\author{
Ei-Ichi Izawa, ${ }^{1,2}$ Gergely Zachar, ${ }^{3}$ Shin Yanagihara, ${ }^{1,2}$ and Toshiya Matsushima ${ }^{1}$ \\ ${ }^{1}$ Graduate School of Bioagricultural Sciences, Nagoya University, Nagoya 464-8601, Japan, ${ }^{2} J a p a n$ Society for Promotion of Science Chiyoda-ku, Tokyo \\ 102-8471, Japan and ${ }^{3}$ Department of Ecology, Institute for Zoology, Szent Istvan University, Budapest H-1400, Hungary
}

Effects of bilateral chemical lesions of the medial basal ganglia [lobus parolfactorius (LPO)] were examined in 7- to 14-d-old domestic chicks. Chicks were trained in a color discrimination task, in which the subject had to peck one of the two colored beads associated with rewards that differed in quantity (amount of food) and/or temporal proximity (delay of food delivery from peck). In experiment 1, food was given without delay, and chicks successfully learned to choose a colored bead that was associated with a larger reward than the other. In experiment 2, a colored bead (red) was associated with a large reward delivered after a delay $(D=1,2$, or $3 \mathrm{sec})$, whereas another (yellow) was associated with a small reward delivered immediately. In intact and sham-operated conditions, chicks with a longer $D$ chose the red bead progressively fewer times. Selective lesions to the caudal LPO (but not the rostral LPO) caused impulsive choice, and the ablated chicks chose the yellow bead and gained a small-immediate reward regardless of $D$. However, when retrained in a null-delay condition $(D=0 \mathrm{sec})$, the lesioned chick chose the red bead again. Ability to associate novel colors with reward was also unimpaired. These results suggest that the LPO may be responsible for the anticipation of reward proximity and involved in a suppression of impulsiveness by which animals seek immediate gains. The present results also indicate a striking similarity in functional roles between the avian LPO and the nucleus accumbens/ventral striatum in mammals.

Key words: basal ganglia; nucleus accumbens; chick; reinforcement learning; reward; anticipation

\section{Introduction}

Despite distinct phylogenetic histories, the structures of the basal ganglia are highly conserved among amniotic vertebrates in terms of connectivities, cytoarchitecture, and chemical neuroanatomy (Marín et al., 1998, Reiner et al., 1998). Birds and mammals have similarly organized reciprocal striatonigro/tegmental connections (Csillag, 1999; Mezey and Csillag, 2002), together with a corticostriatothalamic loop. The avian basal ganglia are composed of lobus parolfactorius (LPO) and paleostriatal complex [paleostriatum augmentatum (PA) and paleostriatum primitivum $(\mathrm{PP})]$, and a consensus has been reached that the LPO and PA constitute a structure homologous to the caudateputamen in mammals. They are thus termed the "striatum," whereas the PP is known as the "dorsal pallidum" (Veenman et al., 1995; Metzger et al., 1996; Durstewitz et al., 1999).

Does such structural homology indicate common functional roles of basal ganglia among amniotes? Recent studies in mammals suggested that basal ganglia are involved in reinforcement learning (Schultz et al., 1997; Redgrave et al., 1999, Spanagel and

\footnotetext{
Received July 22, 2002; revised Dec. 9, 2002; accepted Dec. 11, 2002.

This study was supported by grants-in-aid for scientific research to T.M. from the Japan Society for the Promotion of Science (Grant 13640677) and from the Takeda Science Foundation. G.Z. was supported by a research fellowship from the Daiko Foundation. We thank S. Mezey and Dr. A. Csillag (Semmelweis University, Budapest, Hungary) for their guidance. Valuable comments by Drs. S. Watanabe (Keio University, Tokyo, Japan) and G. Vallortigara (University of Trieste, Trieste, Italy) are also acknowledged.

Correspondence should be addressed to Toshiya Matsushima at the above address. E-mail: matusima@ agr.nagoya-u.ac.jp.

S. Yanagihara's present address: Institute of Neuroscience, University of Oregon, Eugene, OR 97403. Copyright $\odot 2003$ Society for Neuroscience $\quad 0270-6474 / 03 / 231894-09 \$ 15.00 / 0$
}

Weiss, 1999; Doya, 2000; Schultz and Dickinson, 2000; Everitt et al., 2001). Neurons in nucleus accumbens (Ac) and caudateputamen responded to reward-associated cue stimuli and are supposed to code anticipation of forthcoming reward (Hikosaka et al., 1989; Apicella et al., 1992; Aosaki et al., 1994; Shidara et al., 1998). The functional roles of the avian basal ganglia have been studied mostly in two learning paradigms, i.e., song learning in the songbird and passive avoidance learning (PAL) in the domestic chick. In the songbird, the LPO constitutes a distinct nucleus (Area X), which forms part of a closed and topographically organized loop called the "anterior forebrain pathway" (AFP) (Doupe and Konishi, 1991; Luo et al., 2001). The AFP is critically involved in the acquisition phases of song learning (Bottjer et al., 1984; Sohrabji et al., 1990; Scharff and Nottebohm, 1991; Doupe and Kuhl, 1999). In domestic chicks, on the other hand, the LPO has been assumed to be a storage site for memory traces (Rose, 1991, 1996). Post-training lesions to the LPO resulted in retrograde amnesia, and chicks failed to avoid a bitter-tasting bead by color cue (Gilbert et al., 1991; Patterson and Rose 1992). Passive avoidance training actually resulted in a series of biochemical and morphological changes in the LPO, ranging from enhanced glucose uptake and expression of adhesion molecules to resulting changes in synaptic ultrastructures (Rose and Stewart, 1999).

To track the evolution of brain and behavior, we developed appetitive paradigms in which chicks were trained to discriminate colored beads associated with food rewards. A population of LPO neurons proved to code chicks' expectancy of forthcoming reward (Yanagihara et al., 2001), suggesting a functional similarity between the LPO and its mammalian counterpart. Corre- 
spondingly, a pretraining lesion interfered with novel reinforcement learning (Izawa et al., 2001, 2002). In this paper we report a novel task in which a post-training lesion of LPO caused drastic changes in behavioral execution per se without any distinct mnemonic deficiencies.

\section{Materials and Methods}

Subjects and apparatus

Experiments were conducted according to the guidelines of the Committee for Animal Experimentation in Nagoya University. A total of 82 domestic chicks (Gallus domesticus, Cobb strain) were used. New hatchlings obtained from fertilized eggs were communally housed in a dark and moisturized breeder at $30^{\circ} \mathrm{C}$ for $\sim 24 \mathrm{hr}$. From day 2, the breeder was illuminated by white light bulbs ( $12 \mathrm{hr}$ light/dark), and individual chicks were housed separately in transparent plastic cages $(28 \times 18 \times 18 \mathrm{~cm})$; chicks had access to food (millet) and water ad libitum until day 5 . On days $6-14$, chicks were fed once a day with 3.7 gm of millet; water was available ad libitum. In all experimental groups, chicks consumed the limited diet within 10-20 min, without showing a sign of reduced feeding motivation even after the LPO lesions. The limited diet did not cause a weight loss, nor did it lead to expression of abnormal behaviors such as hyperaggressiveness or stereotypy (turning around and pecking repetitively). A thermo-controlled chamber $\left(19 \times 33 \times 35 \mathrm{~cm}, 27-30^{\circ} \mathrm{C}\right.$, illuminated by a $25 \mathrm{~W}$ white light bulb) was used for training and tests. A sidewall of the chamber was equipped with a pair of small holes for bead presentation (located side by side, $3 \mathrm{~cm}$ apart) and a millet-dispenser (located at $1.5 \mathrm{~cm}$ below the holes). The colored beads were protruded through the holes as cue signals, and the dispenser delivered one or six pellets of millet ( $\sim 3.1 \mathrm{mg}$ per pellet) as a reward; cues and rewards were controlled by a micro-robot (RCX 1.0, Lego Co.). The behavior of the chick was observed through a one-way window in the chamber.

\section{Behavioral procedures}

A choice task with delayed reward was adopted (Figs. 1A, 2A). A pair of colored beads (cue 1 and cue 2) was simultaneously presented for up to $1.5 \mathrm{sec}$, and the chick chose one of these beads by a single peck. Both beads were immediately withdrawn after pecking, and the chick was not rewarded or rewarded with either one or six pellets of millet after a delay $=\Delta+D$, according to the choice. Here, $\Delta$ is a constant lag of 0.16 sec because of the mechanical constraints (such as bead withdrawal and pellet delivery), and $D$ is an experimental variable.

In experiment 1 (Fig. $1 A$ ), chicks were trained to discriminate colored beads on the basis of the quantity of the associated reward. A blue bead was always associated with no reward ( - , whereas yellow and red beads were associated with one or six pellets. We made three nonoverlapping groups of chicks, i.e., a 6-vs-1 group, a 6-vs-6 group, and a 1-vs-6 group. In the 6 -vs- 1 group ( $n=10$ chicks), the red bead was associated with 6 pellets, whereas the yellow bead was associated with 1 pellet. In the 1-vs-6 group $(n=7)$, the association between the color and the reward was reversed, to avoid a possibility of innate bias for colors. In the 6-vs-6 group $(n=8)$, both the red and the yellow beads were associated with six pellets. The variable $D$ was set to 0 sec in all three groups.

In experiment 2 (Fig. $2 \mathrm{~A}$ ), chicks were trained to discriminate colored beads on the basis of the proximity and quantity of the associated reward. A blue bead was always associated with no reward $(-)$, a yellow bead was associated with one pellet $(D=0 \mathrm{sec})$, and a red bead was associated with six pellets. For the red bead, the variable $D$ differed as $0,1,2$, or $3 \mathrm{sec}$ in different nonoverlapping groups of chicks (Fig. 2Ab). Accordingly, we thus made 10 groups of chicks: 4 groups of sham and 6 groups of lesioned chicks. Of these six lesion groups, four groups received bilateral lesion of the caudal part of LPO (cLPO), and the other two groups received bilateral lesion of the rostral part of LPO (rLPO). Experiment 2 consisted of four phases (Fig. 2B): (1) preoperative training and test (day 7-9), (2) postoperative training and test (day 11-12), (3) null-delay training and test (day 12-13), and (4) novel training and test (day 13-14). Experiment 1 consisted only of the preoperative phase: training on day 7-9 and test on day 9.

Preoperative training and test. After initial acclimation and conditioning, the chicks were trained to choose one of two simultaneously pre-

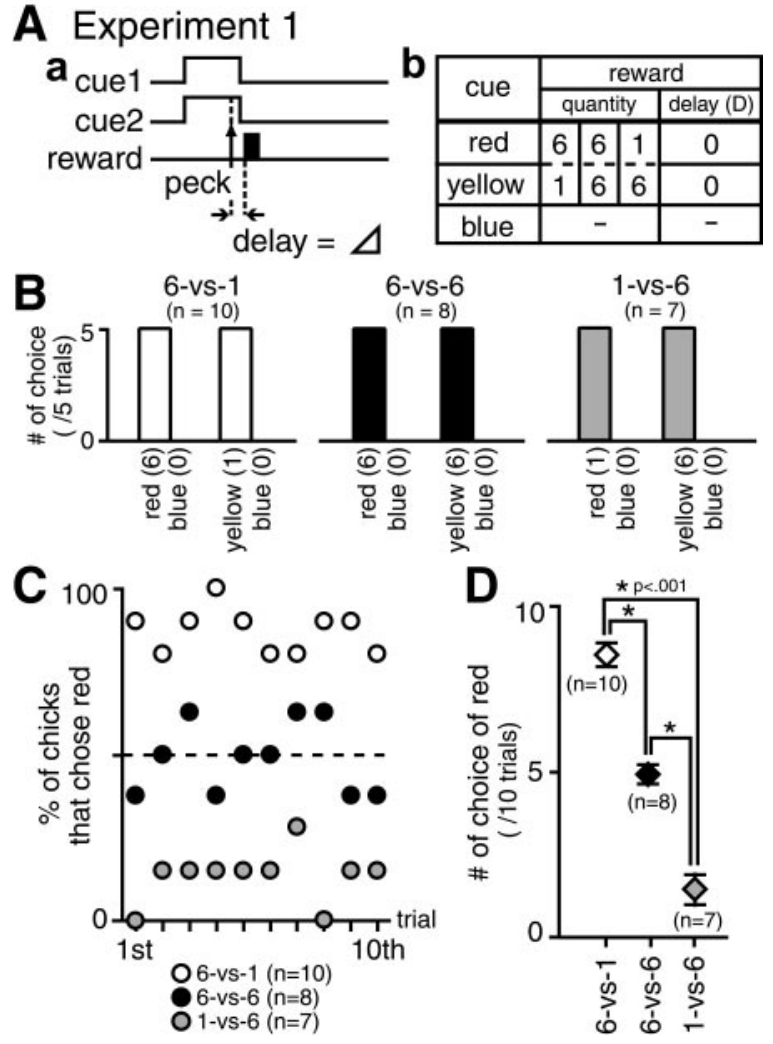

Figure 1. Procedure and results of experiment 1. A, Protocol of the choice task with different reward quantities. In training, three types of trials were given in pseudorandom sequences: trials with a pair of identical blue beads $(-$, no reward), trials with a pair of yellow (1 or 6 pellets) and blue ( - , no reward) beads, and trials with a pair of red ( 6 or 1 pellets) and blue ( - , no reward) beads. The side of presentation was randomized. Chicks thus learned to choose a rewarding color (red or yellow) in the red/blue and the yellow/blue trials and not to peck beads in the blue/blue trials. Chicks were then tested in four trial types that were arranged randomly, i.e., blue/blue (20 trials), yellow/blue ( 5 trials), red/blue ( 5 trials), and red/yellow ( 10 trials). The side of presentation was also randomized. $B$, Number of choices in test trials with red/blue pair and yellow/blue pair; data obtained from the 6 -vs- 1 chicks (open columns), the 6 -vs- 6 chicks (filled columns), and the 1-vs-6 chicks (shaded columns). The number of choices per five trials was averaged over chicks. All of the chicks chose red or yellow; $n$ denotes number of chicks. $C$, Proportion (percentage) of chicks that chose red was plotted along the 10 test trials with red/ yellow pairs. Data were obtained from the 6-vs- 1 chicks (open symbols), the 6-vs-6 chicks ( filled symbols), and the 1-vs- 6 chicks (shaded symbols). D, The number of choices of red was counted in the red/yellow test trials, averaged over chicks, and compared among 6-vs-1, 6-vs-6, and 1 -vs- 6 chicks; post hoc multiple comparisons between pairs among these three groups revealed significant differences at $p<0.001$ (asterisks). Error bars denote SEM.

sented colored beads to gain a reward (Fig. $2 A b$ ). Three types of trials were given in prescheduled random sequences (Fig. $2 \mathrm{Ba}$ ); intertrial intervals were set at $20-30 \mathrm{sec}$. A total of 24 trials ( 12 trials with a blue/blue pair, 6 trials with a yellow/blue pair, and 6 trials with a red/blue pair) constituted one session. The side of the bead presentation was randomized in a balanced manner.

In an initial pilot experiment (data are not included in Results), we trained chicks only with the red/blue and the yellow/blue trials and omitted the nonrewarding trials using a pair of blue/blue. Without the nonrewarding trials, however, choices in the red/blue and yellow/blue trials became blurred and inconsistent, and the number of choices was often distributed around the chance level (i.e., half of the trials). On the other hand, an introduction of the nonrewarding trials enhanced the chick's discrimination, probably because of a reduced probability of gaining rewards. Without the nonrewarding trials, even a subject that pecked randomly would gain a large reward in half of the trials. On the other hand, with a considerable amount of nonrewarding trials in a session, a random pecker would gain a large reward in fewer trials, whereas a dis- 


\section{A Experiment-2}

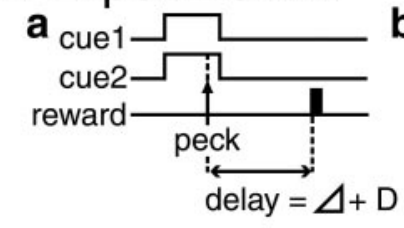

\begin{tabular}{|c|c|c|c|c|c|}
\hline \multirow{2}{*}{ cue } & \multicolumn{4}{|c|}{ reward } \\
\cline { 2 - 5 } & quantity & \multicolumn{3}{|c|}{ delay (D) } \\
\hline red & 6 & 0 & - & 2 & 3 \\
\hline yellow & 1 & 0 & 0 & 0 & 0 \\
\hline blue & - & \multicolumn{4}{|c}{-} \\
\hline
\end{tabular}

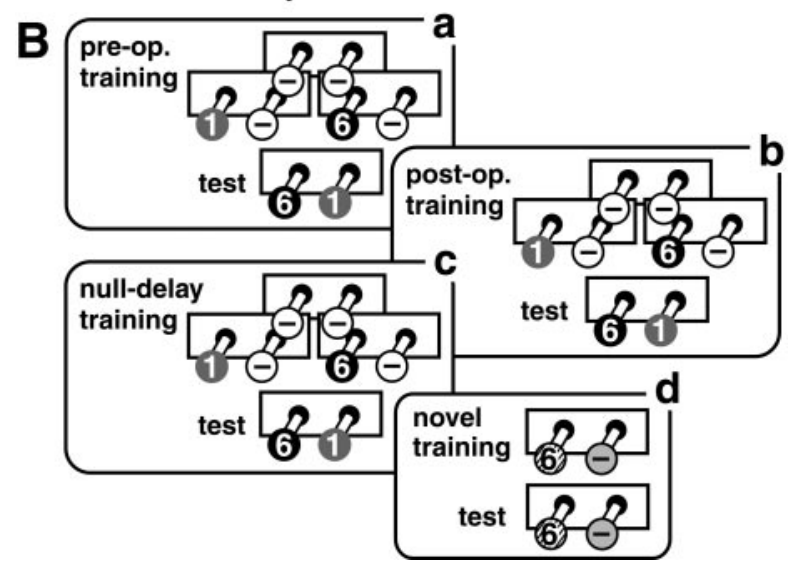

Figure 2. Procedure of experiment 2. A, Protocol of the choice task with delayed reward. $B$, Schedule of training and tests. $B a$, Chicks were trained according to the same protocol as in experiment 1 (Fig. $1 \mathrm{~A}$ ) except that the yellow bead was associated with a reward of one pellet and a longer delay time $(D=0,1,2$, or $3 \mathrm{sec}$ ) was introduced for the red bead. Afterward, four types of trials were given, and chicks were tested with a choice between red (6) and yellow ( 1 ) (preoperative test). $B b$, After recovery from the operation, chicks were retrained and tested according to the same procedures (postoperative test). We examined whether the lesion changed the choice between red (large-late reward) and yellow (small-immediate reward). $B C$, Chicks were further retrained and tested in the null-delay condition, where $D=0$ for both red and yellow. We examined the ability to choose the large reward by color cues. $B d$, Finally, chicks were trained using a novel color pair, e.g., green associated with six pellets (6) and black with no reward $(-)$ with $D=0$ sec for both colors; the ability to associate novel colors with reward was thus examined. In $B a-B c$, symbols indicate the colors of the beads: black $=$ red bead, gray $=$ yellow bead, white $=$ blue bead, respectively. $\ln B d$, black and green beads were used; see Materials and Methods for further explanation.

criminating pecker would be favored. We therefore set the proportion of nonrewarding trials to be $50 \%$ of the total trials in each session in this and the following training and test phases.

Three training sessions were given on day 7 , four sessions on day 8 , and two sessions on day 9. Response was defined as correct when the chick made no peck in the blue/blue trial and when the chick pecked at the rewarding color (red or yellow) in the red/blue or yellow/blue trial, respectively. An incorrect response was followed by another correction trial of the same type. Chicks were rejected if they showed incorrect responses in $\geq 10 \%$ of trials in either one of the trial types during the two sessions on day 9 (i.e., two or more in 12 red/blue trials, two or more in 12 yellow/ blue trials, or three or more in 24 blue/blue trials); 0 and 3 chicks were thus discarded in experiments 1 and 2, respectively. Thereafter, chicks were tested in the fourth type of trial, in which the chick had to choose between red and yellow. We thus examined whether chicks would discriminate between red and yellow to gain a larger reward (experiment 1) and whether the choice of red would differ among groups with different delay time $D$ (experiment 2). A test session was composed of a total of 40 trials given randomly; i.e., 10 trials with red/yellow pair, 5 trials with red/blue pair, 5 trials with yellow/blue pair, and 20 trials with blue/blue pair. Correct responses were similarly rewarded, although correction trials were not given after incorrect responses during the test session. Each chick received either a lesion or a sham operation $\sim 3 \mathrm{hr}$ after the end of the test session on day 9.

Postoperative training and test. After a complete recovery from general effects of the surgery, i.e., at $\sim 36 \mathrm{hr}$ after the operation, the operated-on chicks were trained in a total of six training sessions (four sessions on day
11 and two sessions on day 12) according to the same protocol as in the preoperative training. Three types of trials (red/blue trials, yellow/blue trials, and blue/blue trials) were given, so that chicks consistently discriminated between the rewarding color (red or yellow) and the nonrewarding color (blue). On day 12, the retrained chicks were tested with the red/yellow trials similarly arranged as in the preoperative test, and we examined the effects of the lesion on the choice between red (large-late reward) and yellow (small-immediate reward). The delay time $D$ was unchanged.

In another pilot experiment (not included in Results), we trained and tested the chicks according to the preoperative protocol and then retested them after a $36 \mathrm{hr}$ pause period without any additional retraining sessions. Red and yellow beads were associated with six pellets and one pellet, respectively, both with $D=0 \mathrm{sec}$. In the retest, however, half of the trained chicks showed significantly lower scores in both the blue/blue and the red/yellow trials; these chicks tended to peck at the bead that happened to be located closer to the chick, suggesting that the pecking was not based on color discrimination. An introduction of postoperative training sessions strongly reduced the occurrence of these incorrect responses and thus were supposed to be necessary to normalize the choice scores in the postoperative tests.

Null-delay training and test. To examine the chick's ability to choose large reward by associated color cues, all groups of sham and lesioned chicks with $D>0$ were further retrained in six sessions of null-delay training (i.e., four sessions on day 12 and two sessions on day 13). In the null-delay condition, the delay time $D$ was set to 0 sec for both red and yellow, whereas the association between color and reward quantity remained unchanged. Chicks were then tested according to the null-delay condition, and we examined whether operated-on chicks would still choose the correct cue (red) to gain a large reward (six pellets).

Novel training and test. To examine the chick's ability to form and recall a novel memory, all groups of chicks were trained using a pair of green and black beads. In this phase, the green bead was associated with a six-pellet reward, and the black bead was associated with no reward in half of the chicks; in the other half, the association was reversed: the black bead was associated with a six-pellet reward, and the green bead was associated with no reward. Nonrewarding trials (e.g., black/black pair in the former group) were not given. The side of the bead presentation was randomized in a balanced manner. In the training, we gave a correction trial when the chick showed an incorrect response, i.e., pecking at the nonrewarding color. The training continued until the chick performed correct responses in all of the latest six successive trials on day 13. We counted the total number of training trials required until the criterion of the consecutive six correct trials was met, together with the number of incorrect trials, as indices for the chick's ability to form a novel memory. On day 14, $\sim 12-18 \mathrm{hr}$ after the training, the chicks were tested in another series of 12 trials without correction trials. We counted the number of correct trials in the 12 test trials as an index for the chick's ability to recall the memorized association.

After the test of the novel color association, as the final step of the experiment, chicks were examined for sensorimotor coordination in their pecking behavior in a free food test. The idea behind this test is that any deficiency in visuomotor coordination might be detected as an impaired acuity in food pecking. We counted the number of pecks required to ingest five pellets and averaged the number over three trials spaced at $30 \mathrm{sec}$ intervals.

\section{Statistical analysis}

Parametric tests were used after Bartlett's test for homogeneity of variance. If not applicable, nonparametric tests were used instead. Comparisons were always made between the data of different chick groups (sham and lesion) in corresponding test trials; we did not compare the data between preoperative and postoperative tests as described above. In cases in which ANOVA yielded significant differences, post hoc multiple comparisons were made. In experiment 1 , to compare the number of choices of red (per $10 \mathrm{red} / \mathrm{yellow}$ trials in the test session), we adopted one-way ANOVA in comparing among the three groups of chicks. In experiment 2 , to compare the number of choices between the cLPO-lesioned chicks and the sham, we adopted two-way ANOVA (group $\times$ delay) with 
repeated-measures on subjects, after Bartlett's test of homogeneity of variance for each of the three phases (preoperative, postoperative, and null-delay test). We also compared data between the rLPO-lesioned chicks and the sham by using the same procedure. For comparisons of the mean percentage of correct responses in the test trials (red/blue, yellow/ blue and blue/blue trials; see Table 1), nonparametric Kruskal-Wallis ANOVA by ranks was adopted among groups in each of the three phases, because homogeneity was not found in variance. To analyze performance in the novel training and tests, we adopted one-way ANOVA with repeated measures on subjects among the three groups of chicks, i.e., cLPO, rLPO, and sham. The significance level was $p<0.05$.

\section{Surgery and histology}

In experiment 2, chicks underwent either a sham or a lesion operation after the preoperative test. Chicks were anesthetized by intraperitoneal injection of ketamine and xylazine $[0.40 \mathrm{ml}$ of a $1: 1$ mixture of $10 \mathrm{mg} / \mathrm{ml}$ ketamine (Sankyo Co.) and $2 \mathrm{mg} / \mathrm{ml}$ xylazine (Sigma, St. Louis, MO)]; supplementary injections $(0.10 \mathrm{ml}$ each) were given to maintain a stable anesthesia. Chicks were then fixed on a stereotaxic apparatus. Skin over the skull surface was incised, and the dura mater was cut to expose the brain. For cLPO lesion $(n=24)$, chicks received a bilateral injection of ibotenic acid solution (Sigma) $(0.38 \mu \mathrm{l} \times 12 \mu \mathrm{g} / \mu \mathrm{l} 0.1 \mathrm{M}$ PBS, $\mathrm{pH}=7.3$ per side) using a $1 \mu \mathrm{l}$ Hamilton syringe with a flat tip. Coordinates of the injection site were $5.0 \mathrm{~mm}$ rostral to the frontoparietal suture, $1.0 \mathrm{~mm}$ lateral from the midline, and $5.5 \mathrm{~mm}$ ventral to the brain surface. For rLPO lesion $(n=10)$, chicks received bilateral injections of $0.32 \mu \mathrm{l}$ of the same ibotenic acid per side. Coordinates were $6.9 \mathrm{~mm}$ rostral to the frontoparietal suture, $0.9 \mathrm{~mm}$ lateral from the midline, and $5.1 \mathrm{~mm}$ ventral to the brain surface. For sham operation $(n=20), 0.38 \mu \mathrm{l}$ PBS was bilaterally injected into the cLPO. A single injection was accomplished at a speed of $0.01 \mu \mathrm{l} / \mathrm{min}$ with an additional $5 \mathrm{~min}$ for diffusion before syringe withdrawal. The incised skull was covered with antibiotic, and the skin flap was fixed using superglue. The operated-on chicks were then housed in the breeder for recovery. Transient depression attributable to the surgical operation disappeared completely within 3-4 hr, and the chicks showed normal postures and locomotor activities and actively consumed the diet without showing any motor deficiencies.

On day 14 (after behavioral experiments), chicks were intraventricularly perfused with a fixative ( $4 \%$ paraformaldehyde in $0.1 \mathrm{M} \mathrm{PB}$ ) under a deep ketamine-xylazine anesthesia. Brains were postfixed for 3-4 d at $4^{\circ} \mathrm{C}$, embedded in yolk, and cut into $50 \mu \mathrm{m}$ frontal sections. Sections were mounted, stained with cresyl violet, and analyzed using a camera lucida and graphic software (NIH image). Volume of the lesioned tissue (in cubic millimeters) was estimated by integrating the ablated areas along a series of frontal sections spaced at every $150 \mu \mathrm{m}$. To compare the lesion size, one-way ANOVA was adopted among the six groups of chicks (four cLPO- and two rLPO-lesioned chicks). Terminology of neural nuclei and definition of their boundaries followed the stereotaxic atlas of chick brain by Kuenzel and Masson (1988).

\section{Results}

\section{Experiment 1: choice between large and small reward}

In one group of chicks (6-vs-1 group; $n=10)$, red and yellow beads were associated with six pellets and one pellet of millet as a reward, respectively. In another group (6-vs-6 group; $n=8$ ), both red and yellow beads were associated with six pellets. In the third group ( 1 -vs-6 group; $n=7$ ), association was reversed, and red and yellow beads were associated with one and six pellets, respectively. The blue bead was associated with no reward in all groups. Trained chicks were tested in direct choice between red and yellow. In all of the red/blue and yellow/blue trials in the tests (Fig. $1 B$ ), chicks of all groups pecked only the rewarding color (red or yellow) and avoided the nonrewarding color (blue). In of the nonrewarding (blue/blue) trials, chicks of both groups did not peck at the bead consistently (data not shown). In the red/ yellow trials in the test (Fig. 1C,D), on the other hand, the 6-vs-1 chicks showed a higher choice for the red bead, whereas the 1-vs-6 chicks showed a lower choice for the red bead in a sym- metrical manner; no bias was found between red and yellow. The proportion (percentage) of chicks that chose red was plotted for the 10 trials with red/yellow pairs $(C)$, and the number of times red was chosen per 10 trials was averaged over the chicks $(D)$. Note that the chicks had been trained only in red/blue, yellow/ blue, and blue/blue trials and thus had not experienced the direct choice between red and yellow. Also note the consistent choice of the red bead from the 1 st test trial to the 10 th. The possibility may thus be rejected that chicks were retrained during the course of test trials and then the choice was reoriented to a specific color. A comparison of the number of times red was chosen between the three groups (Fig. $1 D$ ) yielded a significant difference in the mean values (one-way ANOVA) $\left(F_{(2,22)}=102.88 ; p<0.001\right)$; further post hoc multiple comparisons revealed significant differences in all pairs among the three groups $\left({ }^{*} p<0.001\right)$.

We can thus reject the idea that the chicks associated color cues (red and yellow) with the pecking operant with equal strength. Instead, reinforcement by a larger reward (larger in quantity) must have formed a stronger association in the training, so that the chicks could immediately choose the appropriate color from the very first choice trial between red and yellow. In experiment 2, we examined whether the other aspect of the reward (the temporal proximity) could change the choice.

\section{Experiment 2: effects of LPO lesions on the choice between large-late reward and small-immediate reward Histology}

Injection of ibotenic acid reliably lesioned localized areas in LPO (Fig. 3). The whole LPO spanned a range of anterior (A) 9.0 to A12.4 according to the chick brain atlas (Kuenzel and Masson 1988), and the lesion in the cLPO groups was located mainly in the caudal part (A9.2-A11.0). The lesion in the rLPO group was comparable in size but located in the rostral part (A10.4-A12.2). Between the right and left LPO, we did not find significantly different lesion sizes in any of the six groups (paired $t$ test; $p$ values ranging from 0.324 to 0.768 ). The sizes of lesion in the bilateral LPOs were thus summated and compared among groups (Table 1). Although the cLPO lesions tended to be larger than the rLPO lesions, statistically significant differences were not found.

The cLPO lesions included the rostral part of nucleus accumbens, although we could not unequivocally delineate the boundary of Ac on the basis of our Nissl-stained specimens. According to the atlas of Kuenzel and Masson (1988), the Ac extends rostrocaudally from A8.2 to A10.0, whereas the cLPO lesion extended from A9.2 to A11.0. On the basis of the distribution of neurons projecting to the ventral tegmental area (striatotegmental neurons) and those projecting to the substantia nigra (striatonigral neurons), Csillag and his colleagues (Mezey and Csillag, 2002) argued that the boundary of the Ac should be relocated far laterally and rostrally, including the medial part of the LPO; the expanded Ac is also coextensive with the medial LPO region with a relatively weak dopamine receptor-related phosphoprotein-like immunoreactivity. The lesion in the cLPO groups included the rostral part of the Ac defined by Kuenzel and Masson (1988) and nearly overlapped with the distribution of the striatotegmental neurons (Mezey and Csillag, 2002). On the other hand, the paleostriatal complex remained intact in all cases. After recovery from the operation, chicks of both groups consumed foods as actively as the sham chicks, without showing any distinctive symptoms of the hypoactivity found in chicks with larger LPO lesions (Izawa et al., 2002); thus general arousal and feeding motivation remained unimpaired. 


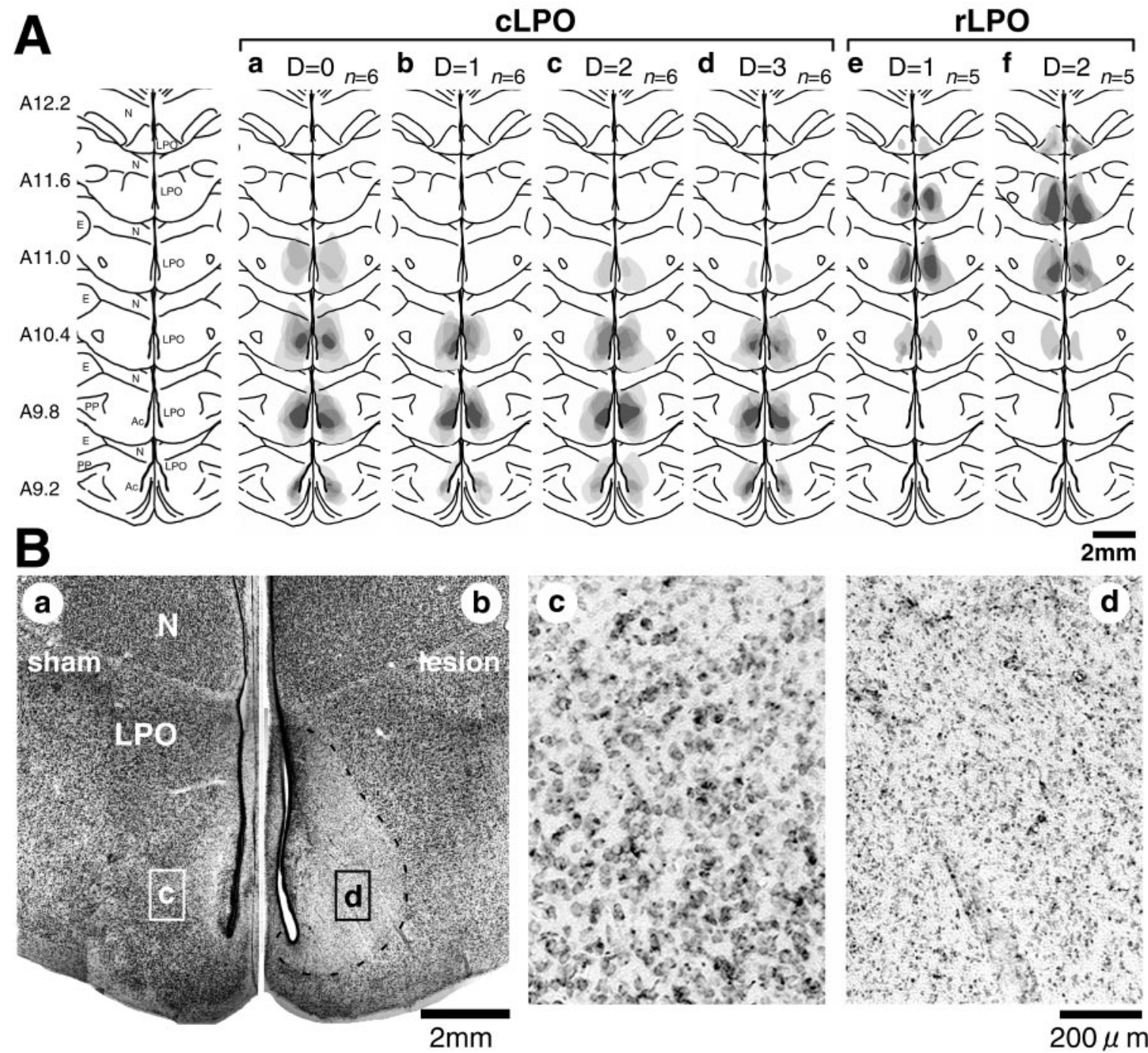

Figure 3. Histological reconstruction of ibotenic acid lesions in six groups of chicks. A, Lesioned areas are superimposed on frontal sections of the telencephalon; the coordinates (A9.2-A12.2) follow the stereotaxic atlas of Kuenzel and Masson (1988). Dark areas indicate overlaps among chicks. a- dindicate four groups of chicks with caudal LPO lesion (CLPO), and e and $f$ indicate two group of chicks with rostral LPO lesion ( $r L P O)$. The CLPO lesions included the rostral part of the nucleus accumbens. Groups differed also in the delay time $(D=0,1,2,3 ;$ see Fig. $2 A) ; n$ denotes the number of chicks in each group. $A c$, Nucleus accumbens; $E$, ectostriatum; $L P O$, lobus parolfactorius; $N$, neostriatum; $P P$, paleostriatum primitivum. $B$, Representative photomicrographs of Nissl-stained caudal LPO in a sham $(a)$ and a lesioned $(b)$ chick; note the clear boundary between lesioned area and its surroundings ( $b$, dashed line). Regions in rectangles marked by c and $d$ are shown at a higher magnification in c and $d$, respectively.

\section{Effects of cLPO lesion}

The cLPO-lesioned chicks showed impulsive choice of smallimmediate reward over late-large reward, with no apparent deficiency in the association between color cues and reward quantity. Choice scores in tests (i.e., number of choices of large reward in the red/yellow trials) were compared between the sham and the cLPO-lesioned chicks (Fig. 4A,B). Comparisons were made among eight groups with corresponding $D$ values, i.e., the four sham groups $(D=0,1,2$, and $3 \mathrm{sec})$ and the four cLPO groups $(D=0,1,2$, and $3 \mathrm{sec})$.

In the preoperative tests, statistic examination using two-way ANOVA revealed no interactions between group and delay $\left(F_{(3,36)}=0.344\right.$; NS), but a significant effect was found in the factor of delay $(D=0,1,2$, and $3 \mathrm{sec})\left(F_{(3,36)}=42.979 ; p<\right.$ $0.0001)$. In the factor of group (cLPO and sham), on the other hand, significant effect was not found $\left(F_{(1,36)}=0.278\right.$; NS). It is therefore concluded that chicks given longer delay times $(D)$ made progressively fewer choices of the red bead associated with the large reward. Scores of the choice performances in the other test trials (red/blue trials, yellow/blue trials, and blue/blue trials) are summarized in Table 1 (preop.); no difference was found among the 10 groups of chicks.

In the postoperative tests, on the other hand, the cLPOlesioned chicks became impulsive. Two-way ANOVA revealed significant interaction in group $\times$ delay $\left(F_{(3,36)}=9.639 ; p<\right.$ 0.0001 ), indicating significantly fewer choices of the large reward in the cLPO-lesion groups than in the sham control chicks with the corresponding delay time $(D)$. Post hoc multiple comparisons between pairs of the sham and the cLPO groups with the same $D$ values showed significant differences $(p<0.01)$ only between the pairs with $D=1$ and $2 \mathrm{sec}$. Both the sham $(D=0 \mathrm{sec})$ and the cLPO $(D=0 \mathrm{sec})$ groups showed high choice scores without a significant difference; thus cLPO lesion did not cause chicks to avoid generally the colored beads associated with large reward. Between the sham $(D=3 \mathrm{sec})$ and the cLPO $(D=3 \mathrm{sec})$, similarly, no statistically significant difference was found. In other trial types, the sham and the cLPO chicks showed high scores of 
Table 1. Lesion sizes and percentage correct response in tests

\begin{tabular}{|c|c|c|c|c|c|c|c|c|c|c|c|c|}
\hline \multirow{2}{*}{ Group } & & \multirow[b]{2}{*}{$n$} & \multirow[b]{2}{*}{ Lesion size $\left(\mathrm{mm}^{3}\right)$} & \multicolumn{3}{|c|}{ Pre-operation } & \multicolumn{3}{|c|}{ Post-operation } & \multicolumn{3}{|c|}{ Null-delay } \\
\hline & & & & $\mathrm{r} / \mathrm{b}$ & $y / b$ & $\mathrm{~b} / \mathrm{b}$ & $\mathrm{r} / \mathrm{b}$ & $y / b$ & $b / b$ & $\mathrm{r} / \mathrm{b}$ & $y / b$ & $\mathrm{~b} / \mathrm{b}$ \\
\hline \multirow{4}{*}{$D=0$} & CLPO & 6 & $3.57 \pm 0.51$ & 100 & 100 & $99.2 \pm 0.83$ & 100 & 100 & $98.3 \pm 1.05$ & NA & NA & NA \\
\hline & Sham & 5 & NA & 100 & 100 & $98.0 \pm 2.00$ & 100 & 100 & $96.0 \pm 1.87$ & NA & NA & NA \\
\hline & CLPO & 6 & $2.41 \pm 0.27$ & 100 & 100 & $96.7 \pm 2.11$ & 100 & 100 & $97.5 \pm 1.71$ & 100 & 100 & $98.3 \pm 1.05$ \\
\hline & Sham & 5 & NA & 100 & 100 & $99.0 \pm 1.00$ & 100 & 100 & $99.0 \pm 1.00$ & 100 & 100 & $98.1 \pm 1.22$ \\
\hline \multirow[t]{3}{*}{$D=1$} & $r L P O$ & 5 & $2.02 \pm 0.30$ & 100 & 100 & $97.0 \pm 2.00$ & 100 & 100 & $97.0 \pm 2.00$ & 100 & 100 & $95.0 \pm 2.24$ \\
\hline & CLPO & 6 & $2.61 \pm 0.31$ & 100 & 100 & $95.8 \pm 1.54$ & 100 & 100 & $98.3 \pm 1.67$ & 100 & 100 & 100 \\
\hline & Sham & 5 & NA & 100 & 100 & $98.0 \pm 2.00$ & 100 & 100 & $99.0 \pm 1.00$ & 100 & 100 & $99.0 \pm 1.00$ \\
\hline \multirow[t]{2}{*}{$D=2$} & $r L P O$ & 5 & $2.07 \pm 0.23$ & 100 & 100 & $95.0 \pm 1.58$ & 100 & 100 & $93.0 \pm 2.55$ & 100 & 100 & $95.0 \pm 2.58$ \\
\hline & CLPO & 6 & $2.97 \pm 0.49$ & 100 & 100 & $96.7 \pm 1.67$ & 100 & 100 & $97.5 \pm 1.71$ & 100 & 100 & $96.7 \pm 1.67$ \\
\hline$D=3$ & Sham & 5 & NA & 100 & 100 & $96.0 \pm 2.45$ & 100 & 100 & $97.0 \pm 2.00$ & 100 & 100 & $99.0 \pm 1.00$ \\
\hline \multicolumn{2}{|c|}{ Statistical values } & & $\begin{array}{l}\text { NS } \\
F_{(5,28)}=2.38^{a}\end{array}$ & & & $\begin{array}{l}\text { NS } \\
H_{(9,54)}=7.06^{b}\end{array}$ & & & $\begin{array}{l}\text { NS } \\
H_{(9,54)}=8.09^{b}\end{array}$ & & & $\begin{array}{l}\text { NS } \\
H_{(7,43)}=9.13^{b}\end{array}$ \\
\hline
\end{tabular}

Performances in the red/yellow trials are separately shown in Fig. 4. Data are shown as mean \pm SEM. NA, Data are not available.

${ }^{a}$ Result of one-way ANOVA is shown with $F$ value.

${ }^{b}$ Results of Kruskal-Wallis test are shown with $H$ values.
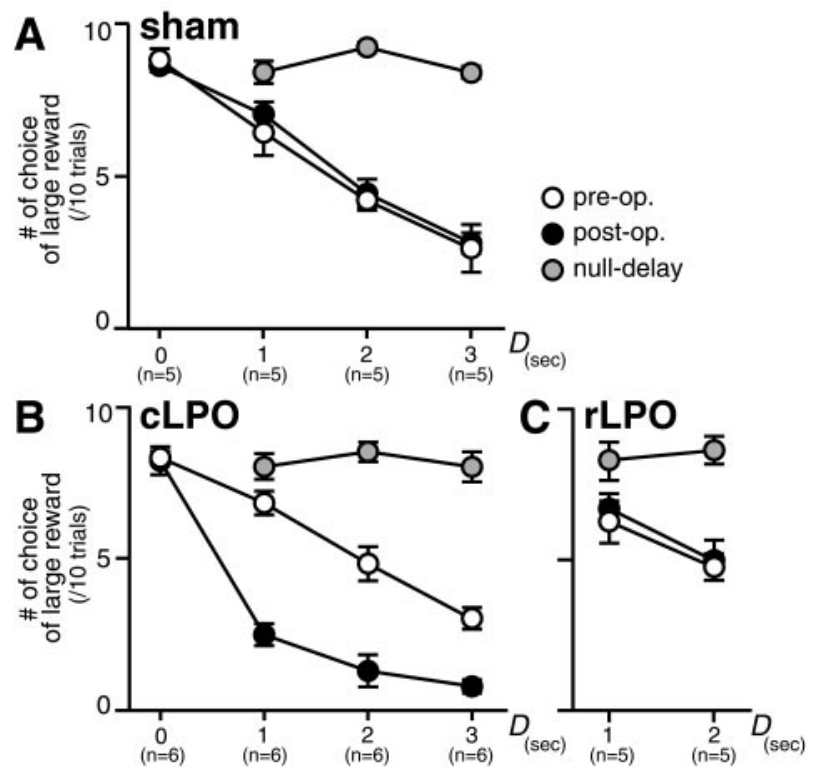

Figure 4. Effects of LPO lesions on the choice between red (large-late reward) and yellow (small-immediate reward). The number of choices for the red bead per 10 trials in the tests was averaged over chicks in each group and plotted against the delay time (D). Error bars denote SEM, and $n=$ number of chicks. $A$, In four groups of sham chicks (sham injection into $\operatorname{LPO} ; D=$ $0,1,2$, or $3 \mathrm{sec}$, respectively), longer $D$ resulted in progressively lower chance of choosing red (large-late reward) in both preoperative and postoperative tests. After retraining in the nulldelay condition, however, chicks of all groups chose red (large reward), suggesting that the association between color cue and reward quantity was unimpaired. $B$, In four other groups of chicks ( $D=0,1,2$, or $3 \mathrm{sec}$, respectively), CLP0 was lesioned as shown in Figure $2 A$. The choice in the postoperative test differed from the preoperative test in three groups of chicks with $D \geq$ $1 \mathrm{sec}$; chicks became impulsive and chose the small-immediate reward. After retraining in the null-delay condition, chicks chose the large reward, indicating that the lesion did not impair the chicks' ability to discriminate colors on the basis of the quantity of the associated reward. C, Two groups of rLPO-lesioned chicks ( $D=1$ and $2 \mathrm{sec}$ ) were trained and tested in the same procedure but remained unimpulsive. Null-delay retraining similarly caused a shift toward large reward. See Results for statistic comparisons.

correct responses without significant differences (Table 1, postop.), suggesting that the cLPO lesion did not impair the learned associations between color cues and the reward.

In the null-delay tests, chicks of all groups chose larger reward regardless of the delay time $(D)$ in the preceding training and tests, indicating that the lesioned chicks were successfully retrained in the null-delay condition. No interaction was found in group $\times$ delay: two-way $\operatorname{ANOVA}\left(F_{(3,36)}=0.073 ;\right.$ NS $)$. One-way ANOVA failed to reveal significant effects of the group factor $\left(F_{(1,36)}=1.694 ; \mathrm{NS}\right)$ or the delay factor $\left(F_{(3,36)}=0.796 ; \mathrm{NS}\right)$. The immediate large reward associated with the red bead was salient enough to reorient the choice in the cLPO-lesioned chicks. In other trials types (Table 1, null-delay), similar to the preoperation and postoperation data, the chicks consistently chose the rewarding color (red or yellow).

\section{Effects of $r L P O$ lesion}

In contrast, rLPO lesion did not cause impulsive choice (Fig. $4 A, C)$. Comparisons were made among four groups with corresponding $D$ values; i.e., the two sham groups $(D=1$ and $2 \mathrm{sec})$ and the two rLPO groups $(D=1$ and $2 \mathrm{sec})$. In the preoperative test, the group of rLPO-lesioned chicks showed comparable choice scores to groups of the sham chicks with the same delay. Two-way ANOVA revealed no interactions between group and delay $\left(F_{(1,16)}=0.212\right.$; NS), but a significant effect was found in the factor of delay $\left(F_{(1,16)}=6.80 ; p=0.019\right)$. In the factor of group, on the other hand, significant effect was not found $\left(F_{(1,16)}=0.024 ; \mathrm{NS}\right)$. In the postoperative test, similarly, a significant effect was found in the factor of delay $\left(F_{(1,16)}=17.6 ; p<\right.$ $0.001)$, but not in the factor of group $\left(F_{(1,16)}=0.145\right.$; NS) without interaction between them $\left(F_{(1,16)}=1.31\right.$; NS). In four chicks [two chicks in the $\operatorname{rLPO}(D=1)$ and two chicks in the $\operatorname{rLPO}(D=$ 2)], the lesion extended as far caudally as A10.4 (Fig. 3A), somewhat overlapping with the cLPO lesions. However, the choice scores in these four chicks ranged around the respective mean values, showing no sign of impulsiveness. We thus conclude that lesion of the rLPO caused no effects on the choice. In the nulldelay tests, no significant effects were found in either factor: delay $\left(F_{(1,16)}=1.60 ; \mathrm{NS}\right)$; group $\left(F_{(1,16)}=0.711 ; \mathrm{NS}\right)$; interaction $\left(F_{(1,16)}=0.178 ; \mathrm{NS}\right)$. The immediate large reward was similarly effective for the rLPO-lesioned chicks as it was for the cLPOlesioned and the sham chicks.

\section{No effects of lesions in novel learning}

Both cLPO and rLPO lesions failed to interfere with either acquisition or recall of novel associations (Fig. 5). The cLPO-lesioned chicks tended to show more trials for criterion, but the difference was not significant in either the total number of training trials until the criteria (Fig. $5 A)\left(F_{(2,51)}=1.481\right.$; NS) or the number of incorrect trials in training $(B)\left(F_{(2,51)}=1.345\right.$; NS) (one-way ANOVA). In the number of correct choices at tests $(C)$, similarly, no significant effect of the group factor was found $\left(F_{(2,51)}=1.284\right.$; NS). 

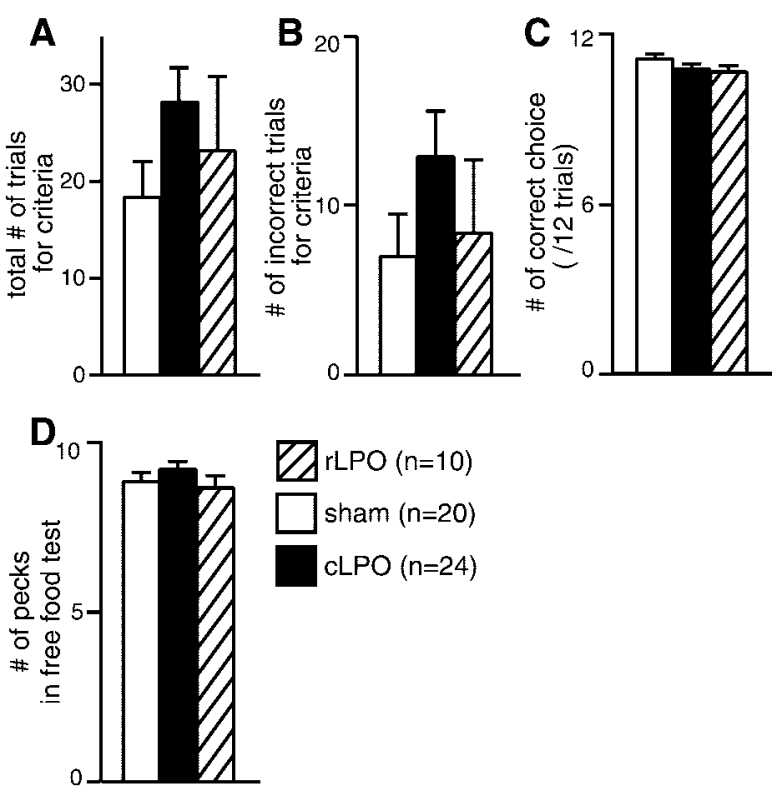

Figure 5. No effects of LPO lesions on novel training. $A, B$, Total number of trials $(A)$ and number of incorrect trials $(B)$ during the novel training procedure (Fig. $2 B d$ ) were compared among three groups of chicks: sham, CLPO-, and rLPO-lesioned chicks. Training continued until chicks met the criteria of correct choice in six successive trials. The CLPO-lesioned chicks tended to have longer training times than others, although no statistically significant difference was found. C, The number of correct choices per 12 test trials was compared, and no significant differences were found among groups. $D$, The number of pecks required for chicks to ingest five pellets showed no significant differences among groups, suggesting that the pecking accuracy was unimpaired. Columns and error bars indicate mean \pm SEM, respectively. One-way ANOVA was adopted after Bartlett's test, both at $p<0.05$.

Finally, the free food test $(D)$ revealed no effects of group factor on the number of pecks $\left(F_{(2,51)}=0.735\right.$; NS). Sensorimotor coordination required for pecking was thus unimpaired.

\section{Discussion}

The present results revealed that 1-week-old chicks are endowed with high cognitive capabilities to associate, recognize, and anticipate events in the external world, thus optimizing their behavioral consequences. The main conclusions drawn from the present study are as follows. First, an association is formed between color cues and reward anticipation and not between color cues and pecking action per se. Actually, in both experiments 1 and 2, chicks were trained to choose between a rewarding color (either red or yellow) and a nonrewarding color (blue) and then tested in a direct choice between the two rewarding colors (red and yellow). Associated rewards differed in quantity (amount of food available) and/or proximity (inverse of the delay time). The results of experiment 1 specifically suggested that chicks anticipated the associated quantity ( six pellets for red and one pellet for yellow), which then led chicks to selective pecking. If pecking was otherwise directly associated with the color cue, and chicks automatically pecked red and yellow beads in the training trials, we would have expected an equal probability of pecking at both red and yellow also in the test trials. This was not the case (Fig. 1C,D), and the choice differed in test trials among the three groups of chicks. The results of experiment 2 (preoperative tests in Fig. 4) further suggest that chicks also anticipated the proximity of the reward.

Second, the process responsible for the anticipation of reward quantity might be dissociable from that for the anticipation of reward proximity. As shown in experiment 2, the cLPO lesion reliably caused impulsive choice in groups trained with delay $(D=1,2,3 \mathrm{sec})$; lesioned chicks tended to peck yellow to gain an immediate-small reward. However, when chicks had been trained without a delay time $(D=0 \mathrm{sec})$ in the preoperative training, the cLPO-lesioned chicks did not differ from the sham chicks in their choice of red in the postoperative tests, suggesting that lesioned chicks still anticipated the quantity. We can reasonably assume that the CLPO is specifically involved in the anticipation of the reward proximity, rather than the quantity.

Third, when localized in smaller areas, the LPO lesions do not cause anterograde amnesia (Fig. 5), in contrast to our previous reports (Izawa et al., 2001, 2002). In previous studies, we made much larger lesions expanding from A9.4 through A12.2 and found that the ablated chicks were severely impaired in novel tasks reinforced by a food/water reward without (Izawa et al., 2001) and with (Izawa et al., 2002) a delay time. We may assume that the rostral and caudal regions of the LPO are functionally differentiated and that both regions are required for novel learning.

Fourth, we could argue that the chick behaved reasonably in choice but did not necessarily optimize its long-term gain. According to most normative theories of behavior, it is assumed that behaviors are organized so that expected net gain is maximized, with increased benefits and decreased costs (Alcock, 2001). Actually, a chick maximizes the immediate gain by choosing a cue color that had been associated with a larger reward (experiment $1)$. However, in a choice between large-late reward and smallimmediate reward (experiment 2), chicks chose the latter and gained smaller reward, although the intertrial interval (20-30 $\mathrm{sec}$ ) was set much longer than the delay time associated with the large reward $(1-3 \mathrm{sec})$. The net gain expected in the whole sequence of trials was thus not maximized. The "impulsiveness" in intact chicks seems conspicuous when compared with rats (Cardinal et al., 2001), which waited for tens of seconds for a larger reward. The possibly predisposed "impulsiveness" in domestic chicks might have some survival benefits in the long term, although such was not evident in this study.

As described in Introduction, LPO lesions are reported to induce retrograde amnesia in PAL, and lesioned chicks failed to avoid a colored bead associated with aversion (Gilbert et al., 1991; Patterson and Rose, 1992). Our present study also revealed, although in the appetitive learning paradigm, that the LPO is involved in the execution of the learned behavior. However, our results are not compatible with the results in PAL in one critical point; namely, the lesion did not impair the memorized association of cue colors in our study, and the enhanced impulsiveness does not account for the retrograde amnesia in PAL. The contradiction might be attributable to the difference in the age, the training methods, the nature of reinforcement, or the method of brain lesion; some of these differences might account for the different results obtained.

Comparison of the present study with mammalian counterparts reveals a striking similarity in functional significance of the ventral striatum. In mammals, it has been shown that lesion or dopamine depletion in Ac severely affects behavioral execution. Most notably, localized lesioning to the Ac core region (but not anterior cingulate cortex and medial prefrontal cortex) caused impulsive choice in rats (Cardinal et al., 2001). Although the effective delay time was much longer in rats $(0-60 \mathrm{sec})$ than chicks $(0-3 \mathrm{sec})$, the effects of Ac core lesions were basically identical to those found in this study, supporting the functional similarity between birds and mammals, although it is difficult to define the chick Ac unequivocally as has been described above. 
Actually, birds and mammals are reported to have comparable neural systems that involve the LPO/ventral striatum as a key element. In the mammalian brain, for example, sensory signals issued from higher sensory cortices are passed on to the amygdala, where the associated biological significance (such as reward and punishment) is evaluated (Ono et al., 1995; Toyomitsu et al., 2002). The amygdala projects to the ventral striatum (including Ac), which leads to premotor cortex for the behavioral execution (Rolls, 2000). In the avian brain, on the other hand, the LPO receives projections from the archistriatum (Arch) (Székely et al., 1994), which has reciprocal connections with the intermediate and medial part of hyperstriatum ventrale (Davies et al., 1997). The IMHV is responsible for acquisition of the imprinting visual memories (Horn 1998) and thus is comparable with the higher visual cortices in primates. We might be able to assume that the IMHV-Arch-LPO pathway has a functional network similar to the higher sensory cortex-amygdala-ventral striatum in primates (Rolls, 2000). Actually, our recent single unit recording (Aoki et al., 2002) revealed a population of Arch neurons that responded to the color cues associated with the reward, suggesting a functional similarity to the mammalian amygdala.

If the LPO is functionally similar to the mammalian Ac, it might also be involved in another aspect of behavioral execution, i.e., evaluation of the associated work cost. In a series of rat studies, dopamine depletion localized in Ac is reported to cause impairments in the performance of a high-effort schedule (Aberman and Salamone, 1999; Salamone et al., 2001). In this task, rats are required to press levers repetitively to a predetermined number (e.g., 5, 20, 100-300 times; referred to as "fixed ratio") to gain a certain reward. Rats with dopamine depletion in Ac performed normally in tasks with a low fixed ratio, although in the high ratio tasks they stopped pressing the lever halfway and failed to gain a food reward. The authors conclude that Ac "may be important for enabling rats to overcome behavioral constraints such as work-related response cost" (Salamone et al., 2001). It will be important to know whether similar dopamine depletion could make chicks avoid sensory cues associated with a higher work cost.

\section{References}

Aberman JE, Salamone JD (1999) Nucleus accumbens dopamine depletions make rats more sensitive to high ratio requirements but do not impair primary food reinforcement. Neuroscience 92:545-552.

Alcock J (2001) Animal behavior: an evolutionary approach, Ed 7. Sunderland, MA: Sinauer.

Aoki N, Izawa E-I, Naito J, Matsushima T (2002) Representation of memorized color in the intermediate ventral archistriatum (amygdala homolog) of domestic chicks. Soc Neurosci Abstr 28:189.13.

Aosaki T, Tsubokawa H, Ishida H, Watanabe K, Graybiel AM, Kimura M (1994) Responses of tonically active neurons in the primate's striatum undergo systematic changes during behavioral sensorimotor conditioning. J Neurosci 14:3969-3984.

Apicella P, Scarnati E, Ljungberg T, Schultz W (1992) Neuronal activity in monkey striatum related to the expectation of predictable environmental events. J Neurophysiol 68:945-960.

Bottjer SW, Miesner EA, Arnold AP (1984) Forebrain lesions disrupt development but not maintenance of song in passerine birds. Science 224:901-903.

Cardinal RN, Pennicott DR, Sugathapala CL, Robbins TW, Everitt BJ (2001) Impulsive choice induced in rats by lesions of the nucleus accumbens core. Science 292:2499-2501.

Csillag A (1999) Striato-telencephalic and striato-tegmental circuits: relevance to learning in domestic chicks. Behav Brain Res 98:227-236.

Davies DC, Csillag A, Székely AD, Kabai P (1997) Efferent connections of the domestic chick archistriatum: a phaseolus lectin anterograde tracing study. J Comp Neurol 389:679-693.

Doupe A, KonishiM (1991) Song-selective auditory circuits in the vocal control system of the zebrafinch. Proc Natl Acad Sci USA 88:11339-11343.

Doupe AJ, Kuhl PK (1999) Birdsong and human speech: common themes and mechanisms. Annu Rev Neurosci 22:567-631.

Doya K (2000) Complementary roles of basal ganglia and cerebellum in learning and motor control. Curr Opin Neurobiol 10:732-739.

Durstewitz D, Kröner S, Güntükün O (1999) The dopaminergic innervation of the avian telencephalon. Prog Neurobiol 59:161-195.

Everitt BJ, Dickinson A, Robbins TW (2001) The neuropsychological basis of addictive behaviour. Brain Res Brain Res Rev 36:129-138.

Gilbert DB, Patterson TA, Rose SPR (1991) Dissociation of brain sites necessary for registration and storage of memory for a one-trial passive avoidance task in the chick. Behav Neurosci 105:553-561.

Hikosaka O, Sakamoto M, Usui S (1989) Functional properties of monkey caudate neurons. III. Activities related to expectation of target and reward. J Neurophysiol 61:814-832.

Horn G (1998) Visual imprinting and the neural mechanisms of recognition memory. Trends Neurosci 21:300-305.

Izawa E-I, Yanagihara S, Atsumi T, Matsushima T (2001) The role of basal ganglia in reinforcement learning and imprinting in domestic chicks. NeuroReport 12:1743-1747.

Izawa E-I, Zachar G, Aoki N, Koga K, Matsushima T (2002) Lesions of ventro-medial basal ganglia impair the reinforcement but not recall of memorized color discrimination in domestic chicks. Behav Brain Res 136:405-414.

Kuenzel WJ, Masson M (1988) A stereotaxic atlas of the brain of the chick (Gallus domesticus). Baltimore: Johns Hopkins.

Luo M, Ding L, Perkel DJ (2001) An avian basal ganglia pathway essential for vocal learning forms a closed topographical loop. J Neurosci 21:6836-6845.

Marín O, Smeets WJAJ, González A (1998) Evolution of the basal ganglia in tetrapods: a new perspective based on recent studies in amphibians. Trends Neurosci 21:487-494.

Metzger M, Jiang S, Wang J, Braun K (1996) Organization of the dopaminergic innervation of forebrain areas relevant to learning: a combined immunohistochemical/retrograde tracing study in the domestic chick. J Comp Neurol 376:1-27.

Mezey S, Csillag A (2002) Selective striatal connections of midbrain dopaminergic nuclei in the chick (Gallus domesticus). Cell Tissue Res 308:35-46.

Ono T, Nishijo H, Uwano T (1995) Amygdala role in conditioned associative learning. Prog Neurobiol 46:401-422.

Patterson TA, Rose SPR (1992) Memory in the chick: multiple cues, distinct brain locations. Behav Neurosci 106:465-470.

Redgrave P, Prescott TJ, Gurney K (1999) Is the short-latency dopamine response too short to signal reward error? Trends Neurosci 22:146-151.

Reiner A, Medina L, Veenman CL (1998) Structural and functional evolution of the basal ganglia in vertebrates. Brain Res Brain Res Rev 28:235-285.

Rolls ET (2000) Précis of the brain and emotion. Behav Brain Sci 23: $177-234$.

Rose SPR (1991) How chicks make memories: the cellular cascade from c-fos to dendritic remodeling. Trends Neurosci 14:390-397.

Rose SPR (1996) Molecular processes in the transition from short to longterm memory. In: Brain processes and memory (Ishikawa K, McGaugh JL, Sakata H, eds), pp 69-78. Amsterdam: Elsevier.

Rose SPR, Stewart MG (1999) Cellular correlates of stages of memory formation in the chick following passive avoidance training. Behav Brain Res 98:237-243.

Salamone JD, Wisniecki A, Carlson BB, Correa M (2001) Nucleus accumbens dopamine depletions make animals highly sensitive to high fixed ratio requirements but do not impair primary food reinforcement. Neuroscience 105:863-870.

Scharff C, Nottebohm F (1991) A comparative study of the behavioral deficits following lesions of various parts of the zebra finch song system: implications for vocal learning. J Neurosci 11:2896-2913.

Schultz W, Dickinson A (2000) Neuronal coding of prediction errors. Annu Rev Neurosci 23:473-500. 
Schultz W, Dayan P, Montague PR (1997) A neural substrate of prediction and reward. Science 275:1593-1599.

Shidara M, Aigner TG, Richmond BJ (1998) Neuronal signals in the monkey ventral striatum related to progress through a predictable series of trials. J Neurosci 18:2613-2625.

Sohrabji F, Nordeen EJ, Nordeen KW (1990) Selective impairment of song learning following lesions of a forebrain nucleus in the juvenile zebra finch. Behav Neural Biol 53:51-63.

Spanagel R, Weiss F (1999) The dopamine hypothesis of reward: past and current status. Trends Neurosci 22:521-527.

Székely AD, Boxer MI, Stewart MG, Csillag A (1994) Connectivity of the lobus parolfactorius of the domestic chicken (Gallus domesticus): an an- terograde and retrograde pathway tracing study. J Comp Neurol 348:374-393

Toyomitsu Y, Nishijo H, Uwano T, Kuratsu J, Ono T (2002) Neuronal responses of the rat amygdala during extinction and reassociation learning in elementary and configural associative tasks. Eur J Neurosci 15:753-768.

Veenman CL, Wild JM, Reiner A (1995) Organization of the avian "corticostriatal" projection system: a retrograde and anterograde pathway tracing study in pigeons. J Comp Neurol 354:87-126.

Yanagihara S, Izawa E-I, Koga K, Matsushima T (2001) Reward-related neuronal activities in the basal ganglia of domestic chicks. NeuroReport 12:1431-1435. 